\title{
Mesenchymal stem cells, Vitamin and Zinc as therapeutic cells and factors of COVID-19
}

\author{
Jamshid Ayatollahi ${ }^{1}$, Seyed Hossein Shahcheraghi ${ }^{1}$, Marzieh Lotfi ${ }^{2,3}$, Mahdie Hamidfar ${ }^{1}$, \\ Mohammad Dehghan Niri ${ }^{4}$, Azam Fazilati ${ }^{5}$ \\ 1 Infectious Diseases Research Center, Shahid Sadoughi Hospital, Shahid Sadoughi University of Medical Sciences, Yazd, Iran \\ 2 Department of Medical Genetics, School of Medicine, Shahid Sadoughi University of medical sciences, Yazd, Iran \\ 3 Abortion Research Center, Reproductive Sciences Institute, Shahid Sadoughi University of Medical Sciences, Yazd, Iran \\ 4 Afshar Hospital, Shahid Sadoughi University of Medical Sciences, Yazd, Iran \\ 5 Shahid Sadoughi Hospital, Shahid Sadoughi University of Medical Sciences, Yazd, Iran
}

\begin{abstract}
*Corresponding Author: Dr. Seyed Hossein Shahcheraghi PhD, Infectious Diseases Research Center, Shahid Sadoughi Hospital, Shahid Sadoughi University of Medical Sciences, Yazd, Iran
\end{abstract}

Email: shahcheraghih@gmail.com; Tel: +00989132531389

2019 Coronavirus disease (COVID-19) occurred in Wuhan City, Central China, and has spread rapidly in many countries in world. COVID-19 is produced by a new coronavirus called the extreme acute respiratory coronavirus syndrome 2 (SARS-CoV2) [Recently identified as the 2019 novel coronavirus (2019nCoV)] [1]. However, there was no official approval of an antiSARS-CoV-2 drug or vaccine due to the lack of sufficient evidence. Scientists are trying to improve COVID-19 by the new drugs and methods [2]

Mesenchymal stem cells (MSCs) are well known to have important anti-inflammatory and immunomodulatory effects. MSCs are very frequent in the placenta and umbilical cord blood. Various studies have also shown that MSC therapy can relieve acute / chronic lung damage and ARDS by blocking immune cell infiltration into pulmonary tissues and pro-inflammatory cytokine production. Moreover, MSCs help reduce lung fibrosis and enhance tissue healing. Other than regular antiviral therapy, therapy of cytokine storm syndromes, ARDS and acute lung injury in patients with severe COVID-19 is important in order to prevent disease progression and to reduce morbidity and mortality. The MSCs may therefore be an effective treatment alternative [1, 3].

It has been confirmed in numerous studies that vitamins of niacin and nicotinamide were very good in decreasing damage in animal prototypes with bleomycin-stimulated lung injury. Vitamin C may also be helpful in decreasingCOVID-19, as it can decrease the intensity of infections in the respiratory tract. It was further reported that vitamin D and vitamin $E$ supplementation could enhance resistance to SARS-CoV-2. So, it may be worth combining vitamin supplements with other treatments in COVID19 patients [4].

Zinc, a trace metal, is needed for the immune system because patients with zinc deficiency had serious immune disorders. Funnily, various studies indicate the lack of sense of taste and smell in the initial stages of COVID-19 patients. Zinc deficiency is well known to be correlated with taste loss, and zinc has favorable impacts in topics with tastes loss. Jointly, zinc deficiency in the COVID-19 infection patients is likely to be correlated with taste and odor reduction in these cases $[5,6]$.

\section{Acknowledgments}

The authors thank from Infectious Diseases Research Center of Shahid Sadoughi University of Medical Sciences. All authors contributed in writing the article.

\section{Conflict of interest: None to declare.}

\section{REFERENCES}

1. $\quad \mathrm{Li} \mathrm{H}$, Liu SM, Yu XH, Tang SL, Tang CK. Coronavirus disease 2019 (COVID-19): current status and future perspective. Int J Antimicrob Agents. 2020:105951.

2. Li H, Zhou $Y$, Zhang $M$, Wang $H$, Zhao $Q$, Liu J. Updated approaches against SARS-CoV-2. Antimicrob Agents Chemother. 2020;64(6).

3. Matthay MA, Goolaerts A, Howard JP, Lee JW. Mesenchymal stem cells for acute lung injury: preclinical evidence. Crit Care Med. 2010;38(10 Suppl):S569.

4. Wang LS, Wang YR, Ye DW, Liu QQ. A review of the 2019 Novel Coronavirus (COVID-19) based on current evidence. Int $J$ Antimicrob Agents. 2020:105948.

5. Keyhan SO, Fallahi HR, Cheshmi B. Dysosmia and dysgeusia due to the 2019 Novel Coronavirus; a hypothesis that needs further investigation. SpringerOpen; 2020.

6. Zhang J, Xie B, Hashimoto K. Current status of potential therapeutic candidates for the COVID-19 crisis. Brain Behav Immun. 2020. 